\title{
Iniciativa Hospital Amigo da Criança, mais de uma década no Brasil: repensando o futuro
}

\author{
More than one decade of the Baby-Friendly Health Care Initiative in Brazil: thinking about the future
}

Joel Alves Lamounier'1, Maria Cândida F. Bouzada², Arlete Moreira de S. Janneu³, Ana Goretti K. Maranhão4, Maria de Fátima M. de Araújo ${ }^{5}$, Graciete Oliveira Vieira ${ }^{6}$, Tatiana de Oliveira Vieira ${ }^{7}$

\section{RESUMO}

Objetivo: Rever a trajetória da Iniciativa Hospital Amigo da Criança (IHAC) no Brasil, sob a orientação de 2004, com a inclusão de novos critérios para credenciamento pelo Ministério da Saúde.

Fontes de dados: Informações obtidas por meio de consulta a relatórios do Ministério da Saúde, Fundo das Nações Unidas para a Infância e artigos publicados sobre IHAC nas bases de dados do Medline, SciELO e Lilacs.

Síntese dos dados: Até maio de 2008, foram credenciados 337 hospitais: 153 no Nordeste, 72 no Sudeste, 54 no Sul, 37 no Centro-oeste e 21 no Norte. Perderam o credenciamento dez hospitais. A partir de dezembro de 2004, o Ministério da Saúde considerou que, para um estabelecimento de saúde receber o título de IHAC, é necessário o cumprimento dos critérios globais estabelecidos, como os "Dez passos para o sucesso do aleitamento materno" e também de dez novos requisitos. A portaria 756/16 inclui: garantia de registros civis a pelo menos $70 \%$ dos recém-nascidos, comprovação do cumprimento da Norma Brasileira de Comercialização de Alimentos para Lactentes e limite de taxas de cesarianas. Nos últimos anos, avaliação e análise dos dados mostram menor número de habilitações de IHAC.

Conclusões: A IHAC é uma experiência de sucesso e contribuiu positivamente para o aumento das taxas de amamentação, junto com outras medidas. Entretanto, deve-se refletir sobre os novos critérios que podem dificultar o processo de obtenção e manutenção do título IHAC no país.

${ }^{1}$ Professor titular de Pediatria da Universidade Federal de Minas Gerais (UFMG), Belo Horizonte, MG, Brasil

2Professora adjunta doutora do Departamento de Pediatria da UFMG e membro da Unidade Neonatal do Hospital das Clínicas da UFMG, Belo Horizonte, MG, Brasil

${ }^{3}$ Coordenadora da Comissão de Implantação da Iniciativa Hospital Amigo da Criança (IHAC) no Hospital das Clínicas da UFMG e enfermeira da Maternidade Oto Cirne do Hospital das Clínicas da UFMG, Belo Horizonte, MG, Brasil

${ }^{4}$ Assessora de Projetos Especiais da Fundação de Ensino e Pesquisa em Ciências da Saúde (Fepecs) da Secretaria de Estado da Saúde (SES) do Distrito Federal, Brasília, DF, Brasil

${ }^{5}$ Gerente de mestrado e doutorado da Escola Superior de Ciências da Saúde da Fepecs da SES do Distrito Federal, Brasília, DF, Brasil
Palavras-chave: aleitamento materno; avaliação de programas; assistência hospitalar; Hospital Amigo Criança.

\section{ABSTRACT}

Objective: Evaluate the Baby-Friendly Health Care Initiative in Brazil under 2004 directions, with the inclusion of new criteria for credentials by the Ministry of Health.

Data sources: Information obtained through consultation of reports of the Ministry of Health, The United Nations Children's Fund (Unicef) and articles related to BabyFriendly Health Care Initiative were retrieved at Medline, SciELO and Lilacs.

Data synthesis: Until May 2008, credentials were given to 337 hospitals: 153 in the Northeast, 72 in the Southeast, 54 in the South, 37 in the Midwest and 21 in the North. Ten hospitals lost the credentials. From December 2004 on, the Ministry of Health considered that to be entitled as a BabyFriendly Hospital, the maternity needed to fulfill "Ten steps for the success of breastfeeding" and ten new requirements. The federal decision number 756/16 included: guarantee of civil registers to at least $70 \%$ of the newly-born infants, adhesion to the Brazilian Norm of Food Commercialization for Suckles and limitation of C-sections. Analysis of the data shows that the number of certifications was reduced during the last years.

Conclusions: Baby-Friendly Health Care Initiative is a successful experience and it has contributed positively for the

${ }^{6}$ Professor adjunto doutor da Universidade Estadual de Feira de Santana (UEFS) e presidente do Departamento Científico de Aleitamento Materno da Sociedade Brasileira de Pediatria, Feira de Santana, BA, Brasil ${ }^{7}$ Professora da UEFS e mestranda em Medicina e Saúde pela Universidade Federal da Bahia (UFBA), Salvador, BA, Brasil

Endereço para correspondência:

Joel Alves Lamounier

Departamento de Pediatria

Avenida Alfredo Balena, 190, 2ํandar, Santa Efigênia

CEP 30130-100 - Belo Horizonte/MG

E-mail: jalamo@medicina.ufmg.br

Recebido em: 10/12/2007

Aprovado em: 23/3/2008 
increase of breastfeeding along with other adopted measures. The new criteria must be reevaluated, since it made the process of certification more difficult in the country.

Key-words: breastfeeding; program evaluation; hospital care; Baby-friendly Healthcare.

\section{Introdução}

Nos últimos anos, ocorreu um aumento da prática de amamentação no Brasil $^{(1)}$. A prevalência do aleitamento materno nas crianças aos seis meses passou de $22 \%$, em 1975, para 69\%, em 1999, segundo dados do Ministério da Saúde ${ }^{(1)}$. Os resultados positivos obtidos nos índices de amamentação refletem o trabalho desenvolvido ao longo dos anos pelos profissionais da saúde nas ações de aleitamento materno. Dentre essas ações, a Iniciativa Hospital Amigo da Criança (IHAC) é considerada uma importante estratégia implementada em várias partes do mundo, com impacto positivo nas taxas de aleitamento materno ${ }^{(1)}$. A IHAC e está estruturada em medidas práticas dos estabelecimentos de saúde (hospitais e maternidades) para a proteção, promoção e apoio ao aleitamento materno. Idealizada em 1990 pela Organização Mundial da Saúde (OMS) e pelo Fundo das Nações Unidas para a Infância (Unicef), tem como objetivo melhorar as práticas hospitalares para aumentar as taxas de amamentaçãa ${ }^{(2,3)}$. A estratégia de atuar junto aos hospitais se deve aos fatores identificados como desfavoráveis à amamentação, especialmente aqueles relacionados com informações errôneas e rotinas hospitalares inadequadas. Pelas normas da IHAC, são distinguidos pela qualidade estabelecimentos de saúde que incorporam em suas rotinas ações de aleitamento materno, com informações adequadas sobre vantagens da amamentação natural e o manejo correto das dificuldades na amamentação.

A mobilização de profissionais de saúde e funcionários de hospitais e maternidades para mudanças de condutas para prevenção do desmame precoce se dá por meio de cursos de capacitação, com base nos "Dez passos para o sucesso do aleitamento materno" preconizado pela OMS/Unicef ${ }^{(2,3)}$. Os dez passos consistem de um elenco de medidas que visam prover informações para gestantes e nutrizes sobre os benefícios e o correto manejo da amamentação. As mulheres, além de ter noções sobre a lactação, são informadas sobre estímulos para produção de leite e soluções para os problemas com a amamentação.
Também são informadas das vantagens do aleitamento e conscientizadas sobre as desvantagens do uso de substitutos do leite materno. Portanto, ao adotar os "Dez passos para o sucesso do aleitamento materno", os hospitais ou maternidades empenham-se em atingir estas metas. Os Hospitais Amigos da Criança (HAC), uma vez qualificados nesta categoria, são considerados referência em aleitamento materno e no atendimento humanizado ao recémnascido ${ }^{(1-4)}$ e passam a atuar como local de capacitação de equipes multiprofissionais da área da saúde.

Para dar início à IHAC, foram escolhidos 12 países, dentre os quais o Brasil, que assumiram um compromisso formal de tornar os dez passos uma realidade em seus hospitais. Como reflexo desta política mundial de incentivo ao aleitamento materno, atualmente mais de 20.000 hospitais são credenciados como HAC em mais de 150 países ${ }^{(4,5)}$. No ano de 1992, o Ministério da Saúde e o Grupo de Defesa da Saúde da Criança, com apoio do Unicef e da Organização Pan-Americana de Saúde (OPAS), deram início a esta iniciativa. A implementação dos dez passos em hospitais e maternidades constituiu uma ação do Programa Nacional de Incentivo ao Aleitamento Materno (PNIAM), que passou a integrar a Área de Saúde da Criança da Secretaria de Políticas de Saúde do Ministério da Saúde, em 1998. Recentemente, no Brasil, foram definidos novos critérios para o credenciamento, além dos dez passos ${ }^{(6)}$. Por conseguinte, o objetivo do atual estudo foi analisar a IHAC no contexto atual, bem como atualizar os dados publicados.

\section{Condições para se tornar HAC}

Ações visando estimular os hospitais e maternidades no país a se tornarem "Amigos da Criança" são coordenadas no Brasil pela Área de Saúde da Criança do Ministério da Saúde. Os estabelecimentos de saúde, para se tornarem HAC, precisam ser submetidos à avaliação com base no cumprimento dos critérios globais de cada um dos "Dez passos para o sucesso do aleitamento materno", assim definidos:

- $\left(1^{\circ}\right)$ Ter uma norma escrita sobre aleitamento materno, que deverá ser rotineiramente transmitida a toda equipe de cuidados de saúde.

- $\left(2^{\circ}\right)$ Treinar toda a equipe de cuidados de saúde, capacitando-a para implementar a referida norma.

- $\left(3^{\circ}\right)$ Informar todas as gestantes sobre as vantagens e o manejo do aleitamento materno.

- $\left(4^{\circ}\right)$ Ajudar as mães a iniciar a amamentação na primeira meia hora após o parto. 
- $\quad\left(5^{\circ}\right)$ Mostrar às mães como amamentar e manter a lactação, mesmo se vierem a ser separadas de seus filhos.

- $\quad\left(6^{\circ}\right)$ Não dar aos recém-nascidos nenhum outro alimento ou bebida além do leite materno, a não ser que seja prescrito pelo médico.

- $\left(7^{\circ}\right)$ Praticar o alojamento conjunto (permitir que mães e bebês permaneçam juntos 24 horas por dia).

- $\left(8^{\circ}\right)$ Encorajar o aleitamento sob livre demanda.

- $\quad\left(9^{\circ}\right)$ Não dar bicos artificiais ou chupetas a crianças amamentadas no peito.

- $\quad\left(10^{\circ}\right)$ Encorajar a formação de grupos de apoio à amamentação, para onde as mães devem ser encaminhadas logo após a alta do hospital ou ambulatório ${ }^{(2,3)}$.

Os hospitais credenciados recebem incentivos financeiros, especificados pela portaria GM/MS no. 1.117 , de 7 de junho de $2004^{(7)}$.

Para uma instituição receber a placa comemorativa de HAC, é preciso obter no mínimo $80 \%$ de aprovação dos critérios globais estabelecidos para cada um dos dez passos. Além disso, cumprir também os dez requisitos estabelecidos na portaria MS n ${ }^{\circ} .756 / 16$, de dezembro de 2004 (Anexo 1) ${ }^{(8)}$. A princípio, alguns requisitos são fáceis de serem cumpridos, como: comprovar cadastramento no Cadastro Nacional dos Estabelecimentos de Saúde; não estar respondendo à sindicância; não ter sido condenado judicialmente nos últimos dois anos em processo relativo à assistência prestada no pré-parto, parto, puerpério e período de internação em unidade neonatal; dispor de profissional capacitado para assistência à mulher e ao recém-nascido no ato do parto; possuir comitê de investigação de óbitos maternos, infantis e fetais; apresentar tempo de permanência hospitalar mínima de 24 horas para o parto normal e de 48 horas para parto cesariano; permitir a presença de acompanhante no alojamento conjunto.

No entanto, dentre os novos requisitos, alguns são mais difíceis de atender como a necessidade de a maternidade garantir o registro civil a pelo menos $70 \%$ dos recém-nascidos. Embora esta condição seja um direito inerente a todo cidadão, não tem comprovada relação de causalidade com o aumento da prática de amamentar. Pode, de fato, ter efeito na redução do número de hospitais já credenciados, bem como no de futuros candidatos.

Outro requisito exige que o hospital comprove o cumprimento da Norma Brasileira de Comercialização de Alimentos para Lactentes e Crianças de Primeira Infância. Este requisito encontra-se delineado nos objetivos da IHAC e, portanto, implícito nos dez passos, em especial nos passos
6 e 9. Deste modo, é necessário elaborar um instrumento apropriado para monitoramento da Norma Brasileira na avaliação do cumprimento deste requisito. É possível que tal exigência seja um fator que dificulta o processo de credenciamento de novos hospitais, bem como a manutenção dos atuais, pois esta ação não se configura como atividade de rotina de um hospital.

Além das acima mencionadas, é uma das exigências a apresentação de taxas de cesarianas de acordo com aquela estabelecida pelo gestor estadual/municipal, tendo como referências as regulamentações do Ministério da Saúde. Nesse sentido, a portaria datada de 9 de janeiro de 2008 reviu a portaria SAS/MS 756, reconhecendo que a tendência crescente das taxas de cesarianas no Brasil tem dificultado a expansão da IHAC. A portaria de 9 de janeiro de 2008 altera ainda a redação do critério 8, permitindo que hospitais com taxas de cesarianas acima das estabelecidas pelo gestor recebam o título, desde que apresentem uma redução dessa prática pelo menos no último ano. Para isso, devem comprovar a adoção de medidas no sentido de atingir as taxas estabelecidas, possibilitando, assim, o seu credenciamento como HAC, o que pode servir de estímulo para reduzir essas altas taxas ${ }^{(6)}$.

Os dados mostram que, nos últimos anos, houve uma queda no número de credenciamentos de hospitais (Gráfico 1). Foram credenciadas somente 35 instituições no período de 2005 até agosto de 2007, sendo 21 hospitais em 2005, oito em 2006 e seis em $2007^{(9)}$. É possível que os novos critérios adotados pelo Ministério da Saúde tenham contribuído para estes resultados. Para muitos hospitais e maternidades, a inclusão de novos requisitos pode representar um grande desafio, devido às suas especificidades locais.

Nos 12 anos de IHAC no Brasil (de 1992 a 2004), chama atenção o pequeno número de hospitais com leitos obstétricos em instituições credenciadas ${ }^{(10)}$. Esses dados alertam para a necessidade de medidas para expandir e manter os HAC no país. Nessa perspectiva, as modificações introduzidas, embora benéficas, podem dificultar o processo de credenciamento e de manutenção destes hospitais na IHAC, visto que as maternidades já credenciadas têm que se adequar a essas novas exigências. Também é importante mencionar que, se o hospital não atingir o mínimo necessário no processo de pré-avaliação ou avaliação global, receberá um certificado de compromisso, no qual é estabelecido um prazo para que as metas sejam alcançadas por meio da adequação dos passos identificados como insuficientes. Após o credenciamento, a instituição IHAC 


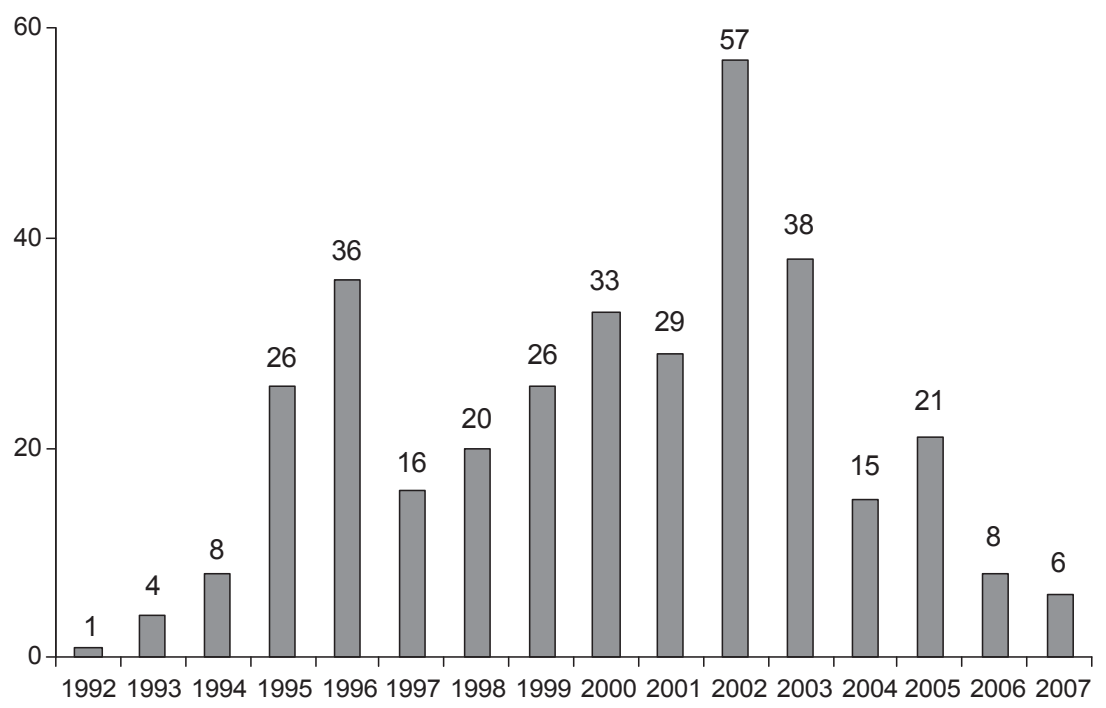

Gráfico 1 - Número de hospitais credenciados como Amigo da Criança no Brasil entre 1992 a agosto de 2007.

passa por reavaliações do Ministério da Saúde a cada três anos e, anualmente, pelas Secretarias de Saúde Estaduais. Caso o hospital não cumpra seis ou mais dos dez passos da IHAC nem os dez requisitos, perde o título de HAC. Como resultado deste procedimento, dez hospitais foram desabilitados oficialmente até agosto de $2007^{(9)}$. Destes, um no Amazonas, um no Ceará, um no Mato Grosso, dois no Maranhão e cinco no Rio Grande do Norte.

\section{Impacto da IHAC no aleitamento materno}

O impacto da IHAC no aumento da duração do aleitamento materno ${ }^{(11)}$, no início precoce do aleitamento ${ }^{(12,13)} \mathrm{e}$ no aleitamento materno exclusivo ${ }^{(5,13,14)}$ tem sido mostrado em algumas pesquisas. Estudo randomizado, incluindo 31 hospitais em Belarus, revelou que a intervenção baseada nos princípios do IHAC aumentou as taxas do aleitamento materno, sobretudo do aleitamento materno exclusivo ${ }^{(5)}$. $\mathrm{Na}$ Escócia, bebês nascidos em hospitais com o status de amigo da criança tiveram $28 \%$ a mais de probabilidade de serem amamentados exclusivamente ao seio do que aqueles que nasceram em outras maternidades ${ }^{(15)}$. Em Boston, um estudo realizado em Unidades de Tratamento Intensivo Neonatal mostrou que as taxas de aleitamento materno na primeira semana, antes da implantação da IHAC em 1995 e após, em 1999, aumentaram de 34,6 para 74,4\%, respectivamente $^{(16)}$. Em países da União Européia, apesar de estudos demonstrarem repercussão positiva da IHAC no aumento das taxas de aleitamento, a Iniciativa é bem desenvolvida somente em três países. Em 19 países, é menor de $15 \%$ a ocorrência dos partos em HAC ${ }^{(17)}$.

Do mesmo modo, no Brasil, foi relatado um significativo aumento das taxas de aleitamento materno e, especialmente, de aleitamento materno exclusivo entre as mães atendidas nos HAC. Bicalho-Mancini e VelásquezMeléndez ${ }^{(18)}$ demonstraram que a taxa de aleitamento materno exclusivo à alta hospitalar em uma unidade hospitalar mudou de 36 para 54,6\%, após aderir à IHAC. Em Londrina, Paraná, depois das mesmas mudanças, verificou-se um aumento nesta taxa de 1,9\% (1994) para 41,7\% (1998). A alimentação exclusiva por fórmulas, que era de 17,9\% em 1994, não mais existia em 1998. Em outra pesquisa, o tempo de aleitamento exclusivo aumentou de 12 para 45 dias $^{(19)}$.

Estudos em Minas Gerais mostram efeitos positivos da IHAC nas taxas de amamentação. A prevalência e a duração do aleitamento materno em Barbacena, Minas Gerais, foram superiores às de outros estudos, especialmente no HAC, revelando a importância da prática dos dez passos ${ }^{(20)}$. Em Montes Claros, a implantação da IHAC em maternidades públicas aumentou significativamente as taxas de aleitamento materno ${ }^{(21)}$. Na Bahia, pesquisa realizada em Feira de Santana observou que a presença de associação entre local de nascimento (hospitais da IHAC) e maiores taxas de aleitamento exclusivo nos primeiros seis meses de vida, aleitamento materno no primeiro ano de vida e menor prevalência de mastite lactacional ${ }^{(22)}$. 
No entanto, vários estudos têm demonstrado que não basta apenas esta abordagem hospitalar para o sucesso do aleitamento materno, sendo necessárias outras formas e estratégias após a alta hospitalar, com formação de grupos de apoio ${ }^{(23)}$. Em Bangladesh, onde 90\% dos bebês nascem no domicílio, a implantação da IHAC da forma proposta não teria efeito. A estratégia foi criar conselheiros comunitários, o que resultou em $70 \%$ de aleitamento materno exclusivo dos lactentes aos cinco meses contra $6 \%$ na área

Tabela 1 - Hospitais Amigo da Criança por Região no Brasil, 2007

\begin{tabular}{lcr}
\hline Região & Número & $\%$ \\
\hline Nordeste & 153 & 46 \\
Sudeste & 72 & 21 \\
Sul & 54 & 16 \\
Centro-oeste & 37 & 11 \\
Norte & 21 & 6 \\
Total & 337 & 100 \\
\hline
\end{tabular}

Fonte: Unicef(27) controle ${ }^{(24)}$. O suporte dos profissionais de saúde é efetivo no prolongamento do aleitamento materno ${ }^{(25,26)}$.

\section{Implantação da IHAC no Brasil}

A primeira instituição de saúde a receber a placa de HAC foi o Instituto Materno-Infantil de Pernambuco (IMIP) em 1992 $2^{(1)}$. Em maio de 2008, dados do Unicef revelaram a existência de 337 instituições credenciadas ${ }^{(9,27)}$, sendo dez estabelecimentos de saúde oficialmente desabilitados até a referida data. Os hospitais estão assim distribuídos por regiões: 153 no Nordeste, 72 no Sudeste, 54 no Sul, 37 no Centro-oeste e 21 no Norte $^{(9)}$ (Tabela 1). A maior parte (46\%) está concentrada na região Nordeste. Roraima, Rondônia e Mato Grosso não contam ainda com nenhum HAC. A distribuição de hospitais por Estados no Brasil está ilustrada na Tabela $2^{(27)}$.

Levando-se em consideração a categoria dos hospitais credenciados, a maioria é público (176/337) ou filantrópico (112/337), sendo apenas 26 hospitais privados e seis

Tabela 2 - Hospitais credenciados Amigo da Criança por Estados e regiões no Brasil até agosto/2007

\begin{tabular}{lcc}
\hline Estado & Região & Número \\
\hline Acre & Norte & 1 \\
Alagoas & Nordeste & 7 \\
Amapá & Norte & 1 \\
Amazonas & Norte & 5 \\
Bahia & Nordeste & 8 \\
Ceará & Nordeste & 37 \\
Distrito Federal & Centro-oeste & 11 \\
Espírito Santo & Sudeste & 4 \\
Goiás & Centro-oeste & 22 \\
Maranhão & Nordeste & 23 \\
Mato Grosso do Sul & Centro-oeste & 4 \\
Minas Gerais & Sudeste & 18 \\
Pará & Norte & 10 \\
Paraíba & Nordeste & 21 \\
Paraná & Sul & 19 \\
Pernambuco & Nordeste & 11 \\
Piauí & Nordeste & 12 \\
Rio de Janeiro & Sudeste & 15 \\
Rio Grande do Norte & Nordeste & 32 \\
Rio Grande do Sul & Sul & 16 \\
Santa Catarina & Sul & 19 \\
São Paulo & Sudeste & 35 \\
Sergipe & Nordeste & 2 \\
Tocantins & Centro-oeste & 4 \\
Total & & 337 \\
\hline Fol Unicer & & \\
\hline
\end{tabular}

Fonte: Unicef(27) 
militares ${ }^{(9)}$. Para conhecer o tipo de gestão dos estabelecimentos de saúde e a natureza de organização, pode-se consultar o Cadastro Nacional de Estabelecimentos de Saúde ${ }^{(28)}$.

Para que as mudanças nas rotinas hospitalares e os dez passos sejam implantados, é preciso amplo apoio e determinação da direção dos hospitais. É fundamental também a confiança de pediatras, obstetras, anestesistas e demais profissionais de saúde nos "Dez passos para o sucesso do aleitamento materno" e empenho para que os mesmos sejam executados.

$\mathrm{Na}$ experiência dos HAC, os resultados desta iniciativa demonstram que o esforço é válido, não só pela humanização do atendimento materno-infantil, mas também pelo aumento das taxas de aleitamento materno exclusivo alcançado com a implantação do programa. Entretanto, são muitas as dificuldades para manter o cumprimento dos dez passos ao longo dos anos ${ }^{(29)}$. O Departamento de Aleitamento Materno da Sociedade Brasileira de Pediatria apóia e incentiva a IHAC no país como uma maneira eficiente de promover, proteger e apoiar a amamentação. Informações sobre a IHAC dirigidas para pediatras e classe médica em geral têm sido publicadas nos últimos anos, destacando a importância desta iniciativa para a promoção do aleitamento materno ${ }^{(1,30)}$.

\section{Superando as dificuldades para implantação da IHAC}

Na implantação de um HAC, podem ocorrer dificuldades de naturezas diversas. Entre os profissionais de saúde, nota-se, em geral, grande resistência inicial à realização das mudanças necessárias no hospital ou maternidade. A maioria dos profissionais de saúde que lida com gestantes, mães e bebês tem pouco conhecimento no manejo da amamentação. Estudos realizados em diferentes países têm encontrado indiferença, inconsistências, atitudes e conhecimentos inadequados por parte dos profissionais de saúde com relação à amamentação( ${ }^{(24,31)}$.

A falta de conhecimentos por parte de médicos, enfermeiras e auxiliares, aliada a atitudes e práticas incorretas no manejo da amamentação, são importantes fatores para o desmame precoce $^{(31,32)}$. Os resultados de diversos estudos mostraram que a maioria dos pediatras entrevistados necessitava de mais informação sobre aleitamento materno e que $72 \%$ não estavam familiarizados com as idéias da IHAC ${ }^{(32)}$. Nos Estados Unidos, Merewood et a ${ }^{(16)}$ ressaltam alguns obstáculos importantes para a implantação da IHAC: a força da indústria de fórmulas infantis, o conhecimento clínico subótimo dos profissionais de saúde e a necessidade de implementar mudanças institucionais significativas. Também existe ainda a crença de que a amamentação natural é algo instintivo e que não necessita ser trabalhada com as parturientes, seus familiares ou mesmo com os próprios profissionais de saúde.

$\mathrm{O}$ uso de bicos, chupetas e mamadeiras em hospitais e maternidades pode indicar pouca convicção no aleitamento materno e dificuldades para o manejo da amamentação. Os bicos e chupetas, além de contribuírem para o desmame, podem ser vistos pela mãe como uma alternativa fácil ao primeiro obstáculo que encontrar após a saída do hospital ${ }^{(33)}$. Portanto, a alta hospitalar precoce também impõe a necessidade de adequado acompanhamento do recém-nascido pelo risco do desmame precoce. Em geral, muitos bebês são desmamados nas primeiras semanas, quando as mães introduzem fórmulas infantis. Embora ocorra um aumento das taxas de aleitamento materno exclusivo nos HAC, essas podem não ser mantidas após a alta hospitalar. Assim, é mais efetiva a combinação da promoção da amamentação no hospital com a manutenção do programa na comunidade, como mostrado no estudo de Coutinho et $a^{(23)}$. De fato, a prevalência do aleitamento materno foi significativamente maior ( $45 \%)$ no grupo que recebeu visitas domiciliares do que no grupo controle (13\%); daí a importância do acompanhamento no domicílio logo após a alta hospitalar, em especial quando realizado por profissionais de saúde.

Assim, é necessária a capacitação dos profissionais de saúde e demais envolvidos com aleitamento materno, dentre os quais voluntários da comunidade das áreas de referência dos hospitais. O trabalho do agente comunitário de saúde, com busca ativa de mãe e filho logo após a alta hospitalar, constitui oportunidade ímpar para identificar os riscos para o desmame precoce e estabelecer medidas de intervenção.

Nesse sentido, importante estratégia para complementar o trabalho desenvolvido durante o pré-natal, pós-parto e após a alta hospitalar é organizar as Unidades e Centros de Saúde na continuidade de ações para o aleitamento materno. As Unidades Básicas de Amamentação necessitam ser estruturadas para que a nutriz seja bem orientada e bem informada e, com isto, mantenha o aleitamento materno exclusivo até os seis meses e complementado até os dois anos ou mais de idade ${ }^{(34,35)}$. Em Belo Horizonte, Minas Gerais, um estudo mostrou que o impacto de ações de promoção do aleitamento materno em um centro de saúde foi positivo na duração e manutenção do aleitamento materno dos dois aos 12 meses de idade ${ }^{(36)}$. 
$\mathrm{O}$ ingresso de um hospital na IHAC significa o reconhecimento ao trabalho desenvolvido pela instituição e constitui referência não só para a comunidade como também para outros hospitais no treinamento de equipes multiplicadoras. A implantação dos dez passos configurase como uma experiência bem sucedida, com impacto positivo na prática da amamentação. No entanto, o atual processo de obtenção e manutenção do título de HAC inclui ações definidas em portaria ministerial (756/16) que podem dificultar e desestimular o credenciamento de novos estabelecimentos.

Vale lembrar que a meta principal da IHAC é a promoção, a proteção e o apoio ao aleitamento materno, uma iniciativa de reconhecido e comprovado sucesso mundial. Entretanto, mais esforços são necessários para aumentar o número de hospitais credenciados no Brasil, o que implica mudanças nas atuais exigências e uma política de maior investimento pelo setor público.

\section{Anexo 1}

Normas para o processo de habilitação do Hospital Amigo da Criança integrante do Sistema Único de Saúde - SUS

A Iniciativa Hospital Amigo da Criança - IHAC no Brasil será desenvolvida consoante às normas e orientações a seguir descritas.

I - O estabelecimento de saúde para ser habilitado pelo gestor estadual/municipal na Iniciativa Hospital Amigo da Criança deverá atender aos seguintes critérios:

1. Comprovar cadastramento no Cadastro Nacional dos Estabelecimentos de Saúde - CNES;

2. Comprovar cumprimento à Norma Brasileira de Comercialização de Alimentos para Lactentes e Crianças na Primeira Infância;

3. Não estar respondendo à sindicância no Sistema Único de Saúde - SUS;

4. Não ter sido condenado judicialmente, nos últimos dois anos, em processo relativo à assistência prestada no préparto, parto, puerpério e período de internação em unidade de cuidados neonatais;

5. Dispor de profissional capacitado para a assistência à mulher e ao recém-nascido no ato do parto;

6. Garantir, a partir da habilitação, que pelo menos $70 \%$ dos recém-nascidos saiam de alta hospitalar com o Registro de Nascimento Civil; comprovado pelo Sistema de Informações hospitalares, mediante incentivo instituído pela Portaria $n^{\circ}$ 938/GM, 20 de maio de 2002;

7. Possuir comitê de investigação de óbitos maternos, infantis e fetais implantado e atuante, que forneça trimestralmente ao setor competente da Secretaria Municipal de Saúde - SMS e/ou da Secretaria Estadual de Saúde - SES as informações epidemiológicas e as iniciativas adotadas para a melhoria na assistência, para análise pelo Comitê Estadual e envio semestral ao Comitê Nacional de Prevenção do Óbito Infantil e fetal;

8. Apresentar taxa percentual de cesarianas conforme a estabelecida pelo gestor estadual/municipal, tendo como referência as regulamentações procedidas do Ministério da Saúde - MS;
9. Apresentar tempo de permanência hospitalar mínima de 24 horas para parto normal e de 48 horas para parto cesariana;

10. Permitir a presença de acompanhante no Alojamento Conjunto;

11. Realizar os "Dez Passos para o Sucesso do Aleitamento Materno", proposto pela Organização Mundial da Saúde e pelo Fundo das Nações Unidas para a Infância, assim definidos:

$1^{\circ}$ ter uma norma escrita sobre aleitamento materno, que deverá ser rotineiramente transmitida a toda equipe de cuidados de saúde;

$2^{\circ}$ treinar toda a equipe de cuidados de saúde, capacitando-a para implementar a referida norma;

$3^{\circ}$ informar todas as gestantes sobre as vantagens e o manejo do aleitamento materno;

$4^{\circ}$ ajudar as mães a iniciar a amamentação na primeira meia hora após o parto;

$5^{\circ}$ mostrar às mães como amamentar e como manter a lactação, mesmo se vierem a serem separadas de seus filhos;

$6^{\circ}$ não dar aos recém-nascidos nenhum outro alimento ou bebida além do leite materno a não ser que seja prescrito pelo médico;

$7^{\circ}$ praticar o alojamento conjunto (permitir que mães e bebês permaneçam juntos 24 horas por dia);

$8^{\circ}$ encorajar o aleitamento sob livre demanda;

$9^{\circ}$ não dar bicos artificiais ou chupetas a crianças amamentadas no peito;

$10^{\circ}$ encorajar a formação de grupos de apoio à amamentação para onde as mães devem ser encaminhadas, logo após a alta do hospital ou ambulatório.

II. O processo de credenciamento/habilitação é iniciado com o preenchimento do questionário de auto-avaliação padronizado pelo Ministério da Saúde e fornecido pela Secretaria de Estado da Saúde - SES, pelo responsável do estabelecimento hospitalar, e encaminhado pela Secretaria Municipal de Saúde - SMS dos municípios não habilitados em Gestão Plena para a SES. 
III. Os municípios habilitados em Gestão Plena e as SES procederão à análise do questionário de auto-avaliação e do cumprimento dos critérios descritos no item I. A SES também designará um avaliador da IHAC para realizar a pré-avaliação do hospital, mediante instrumento padronizado pelo Ministério da Saúde - MS.

IV. Durante o processo de pré-avaliação o estabelecimento de saúde que não atender integralmente aos "Dez Passos para o Sucesso do Aleitamento Materno”, a SMS/SES estabelecerá o prazo para adequação ao cumprimento dos passos pendentes e reavaliação.

V. A partir da verificação do cumprimento dos critérios descritos no item I, o gestor municipal/estadual, dependendo das prerrogativas compatíveis com o nível de gestão, solicitará a avaliação global pela Secretaria de Atenção à Saúde - SAS/MS, mediante o envio de declaração e de cópia dos documentos comprobatórios do cumprimento, solicitando ao Departamento de Ações Programáticas Estratégicas - DAPES/SAS/MS a habilitação do estabelecimento de saúde como Hospital Amigo da Criança, assumindo desta forma, as despesas adicionais decorrentes da habilitação. Após aprovado pela área técnica do DAPES, o resultado será encaminhado ao Gabinete da SAS que deverá providenciar Portaria com a habilitação do estabelecimento devidamente identificado com os seus números no Cadastro Nacional de Estabelecimento de Saúde - CNES. Competirá a Coordenação-Geral dos Sistemas de Informação - CGSI, do Departamento de Regulação, Avaliação e Controle de Sistemas - DRAC/SAS/MS garantir que os sistemas de informação obedecerão às habilitações aprovadas.

VI. A avaliação global dos Dez Passos será realizada por uma equipe de dois avaliadores credenciados e designados pela SAS/MS, sendo um do próprio estado. Os resultados dessa avaliação deverão ser encaminhados pelos avaliadores à referida Secretaria para fins de análise e divulgação.
VII.O hospital que cumprir o estabelecido nesta Portaria receberá a placa de IHAC em solenidade oficial.

VIII.As reavaliações dos hospitais serão realizadas pelo Departamento de Ações Programáticas Estratégicas - DAPES/ SAS/MS - Área Técnica de Saúde da Criança e Aleitamento Materno, a cada três anos ou quando houver denúncia de irregularidades. O hospital poderá ser descredenciado/ desabilitado caso seja constatado o não cumprimento dos critérios e dos Dez Passos listados no item I.

IX. O descredenciamento/desabilitação será feito mediante publicação de portaria revogando o ato anterior de habilitação, editada pela Secretaria de Atenção à Saúde/SAS/MS.

X. As supervisões anuais nos hospitais credenciados serão realizadas pelos avaliadores designados pelas SMS/SES, utilizando como roteiro formulários específicos da IHAC. Os resultados deverão ser encaminhados, até o último dia útil do mês de novembro de cada ano, ao DAPES/SAS/MS - Área Técnica de Saúde da Criança e Aleitamento Materno.

XI. Para a avaliação e a reavaliação globais, a equipe de avaliadores designados contará com dois profissionais de saúde não envolvidos com o treinamento nem com o processo de credenciamento, sendo que um deles não deverá ser do município onde esteja ocorrendo tal processo.

XII.As SES e SMS e os estabelecimentos de saúde credenciados deverão zelar pela continuidade das ações de promoção, proteção e apoio ao aleitamento materno, mantendo o cumprimento dos critérios e dos "Dez Passos para o Sucesso do Aleitamento Materno".

XIII.Os valores de remuneração dos hospitais habilitados como Amigo da Criança constam da Portaria GM/MS nº. 1117, de 07 de junho de 2004. 


\section{Referências bibliográficas}

1. Lamounier JA, Bouzada MC, Janneau AM. Iniciativa Hospital Amigo da Criança em Minas Gerais: situação atual. Rev Med Minas Gerais 2005;15 (Suppl 1):S1-7.

2. World Health Organization/United Nations Children's Fund (WHO/Unicef). Innocenti Declaration on the protection, promotion and support of breastfeeding. Meeting "Breast-feeding in the 1990s: A global initiative". Florence/ Italy: World Health Organization; 1990.

3. United Nations Children's Fund. Baby-friendly Hospital Initiative. $2^{\text {nd }} e d$. New York: Unicef; 2004.

4. United Nations Children's Fund [homepage on the Internet]. 15 years after Innocenti Declaration, breastfeeding saving six million lives annually [cited 2007 Apr 23]. Available from: http://www.unicef.org/media/media_30011.html

5. Kramer MS, Chalmers B, Hodnett ED, Sevkovskaya Z, Dzikovich I, Shapiro $\mathrm{S}$ et al. Promotion of breastfeeding intervention trial (PROBIT): a randomized trial in the Republic of Belarus. JAMA 2001;285:413-20.

6. Brasil - Ministério da Saúde. Secretaria de Atenção à Saúde. Portaria n 9 de 10 de janeiro de 2008. Brasília: Ministério da Saúde, 2008.

7. Brasil - Ministério da Saúde. Secretaria de Atenção à Saúde. Portaria no 1117 de 7 de junho de 2004. Brasília: Ministério da Saúde, 2004.

8. Brasil - Ministério da Saúde. Secretaria de Atenção à Saúde. Portaria n ${ }^{\circ} 756$ de 16 de Dezembro de 2004. Brasília: Ministério da Saúde, 2004. Available from: http://portal.saude.gov.br/portal/arquivos/pdf/Port\%20\%20756\%20.pdf

9. Brasil - Ministério da Saúde [homepage on the Internet]. Brasil: informações de saúde - IHAC [cited 2008 Mar 19]. Available from: http://portal.saude.gov. $\mathrm{br} /$ portal/saude/cidadao/visualizar_texto.cfm?idtxt=24229

10. Araújo MFM, Schmitz BAS. Doze anos de evolução da Iniciativa Hospital Amigo da Criança no Brasil. Rev Panam Salud Publica 2007;22:91-9.

11. Merten S, Dratva J, Ackermann-Liebrich U. Do baby-friendly hospitals influence breastfeeding duration on a national level? Pediatrics 2005;116:702-8.

12. Philipp BL, Merewood A, Miller LW, Chawla N, Murphy-Smith MM, Gomes JS et al. Baby-friendly hospital initiative improves breastfeeding initiation rates in a US hospital setting. Pediatrics 2001;108:677-81.

13. Merewood A, Mehta SD, Chamberlain LB, Philipp BL, Bauchner H. Breastfeeding Rates in US Baby-Friendly Hospitals: Results of a National Survey. Pediatrics 2005;116:628-34.

14. Alam MU, Rahman M, Rahman F. Effectiveness of baby friendly hospital initiative on the promotion of exclusive breast feeding among the Dhaka city dwellers in Bangladesh. Mymensingh Med J 2002;11:94-9.

15. Broadfoot M, Britten J, Tappin DM, MacKenzie JM. The Baby Friendly Initiative and breast feeding rates in Scotland. Arch Dis Child Fetal Neonatal Ed 2005;90:F114-6.

16. Merewood A, Philipp BL. Implementing change: becoming baby-friendly in an inner city hospital. Birth 2001;28:36-40.

17. Cattaneo A, Buzzetti R. Effect on rates of breast feeding of training for the baby friendly hospital initiative. BMJ 2001;323:1358-62.

18. Bicalho-Mancini PG, Velásquez-Meléndez G. Exclusive breastfeeding at the point of discharge of high-risk newborns at a Neonatal Intensive Care Unit and the factors associated with this practice. J Pediatr (Rio J) 2004;80:241-8.
19. Vannuchi MT, Monteiro CA, Rea MF, Andrade SM, Matsuo T. The Baby-Friendly Hospital Initiative and breastfeeding in a neonatal unit. Rev Saude Publica 2004;38:422-8.

20. Ferreira JRT. Aleitamento materno exclusivo até o sexto mês em Barbacena, Minas Gerais: estudo comparativo entre Hospital Amigo da Criança e hospital convencional [tese de mestrado]. Belo Horizonte (MG): UFMG; 2005.

21. Caldeira AP, Gonçalves E. Assessment of the impact of implementing the Baby-Friendly Hospital Initiative. J Pediatr (Rio J) 2007;83:127-32.

22. Vieira GO, Silva LR, Mendes CMC, Vieira TO. Lactational mastitis and babyfriendly hospital initiative, Feira de Santana, Bahia, Brazil. Cad Saude Publica 2006;22:1193-200.

23. Coutinho SB, de Lira PI, de Carvalho Lima M, Ashworth A. Comparison of the effect of two systems for the promotion of exclusive breastfeeding. Lancet 2005;366:1094-100.

24. Haider R, Kabir I, Huttly SR, Ashworth A. Training peer counselors to promote and support exclusive breastfeeding in Bangladesh. J Hum Lact 2002;18:7-12.

25. Bonuck KA, Trombley M, Freeman K, McKee D. Randomized, controlled trial of prenatal and postnatal lactation consultant intervention on duration and intensity of breastfeeding up to 12 months. Pediatrics 2005;116:1413-26.

26. Britton C, McCormick FM, Renfrew MJ, Wade A, King SE. Support for breastfeeding mothers. Cochrane Database of Systematic Reviews 2007; 1 [cited 2007 Jan 24]. Available from: http://www.cochrane.org/reviews/en/ab001141.html/

27. United Nations Children's Fund [homepage on the Internet]. Placar dos Hospitais Amigos da Criança [cited 2008 Mai 23]. Available from: http://www. unicef.org/brazil/pt/activities_9997.htm

28. Brasil - Ministério da Saúde - Secretaria de Atenção à Saúde [homepage on the Internet]. Cadastro Nacional de Estabelecimentos de Saúde [cited 2008 Mar 19]. Available from: http://cnes.datasus.gov.br/Index.asp?Configuracao= 1024\&bro=Microsoft $\% 20$ Internet $\% 20$ Explorer

29. Araújo MF, Schmitz BA. Reassessment of Baby-friendly Hospitals in Brazil. J Hum Lact 2007;23:246-52.

30. Lamounier JA, Maranhão AGK, Moura MF. Iniciativa Hospital Amigo da Criança. In: Rego JD, editor. Aleitamento materno. São Paulo: Atheneu; 2006. p. 401-14.

31. Okolo SN, Ogbonna C. Knowledge, attitude and practice of health worker in Keffi local government hospitals regarding Baby-Friendly Hospital Initiative (BFHI) practices. Eur J Clin Nutr 2002;56:438-41.

32. Schanler RJ, O'Connor KG, Lawrence RA. Pediatricians' practices and attitudes regarding breastfeeding promotion. Pediatrics 1999;103:E35.

33. Silveira FJF, Lamounier JA. Influência do uso da chupeta e do tabagismo materno na amamentação. Rev Med Minas Gerais 2003;13:120-7.

34. Oliveira MI, Camacho LA. Impact of primary health care units' practice on the duration of exclusive breastfeeding. Rev Bras Epidemiol 2002;5:41-51.

35. Oliveira MIC, Gomes MA. As unidades básicas amigas da amamentação: uma nova tática no apoio ao aleitamento materno. In: Rego JD, editor. Aleitamento materno. São Paulo: Atheneu; 2006. p. 415-44.

36. Lana AP, Lamounier JA, César CC. The impact of a breastfeeding promotion program at a health center. J Pediatr (Rio J) 2004;80:235-40. 